\section{Matter-Robotic Calibration for Bioshotcrete} digital matter, iterative calibration.

HOW TO CITE

Bravo, Maite, Stephanie Chaltiel and Wilfredo Carazas. 2018. "Matter-Robotic Calibration for Bioshotcrete".
Temes de disseny 34:80-91.

CASE STUDY

Construction techniques associated with traditional raw earth architecture are characterised by laborious manual tasks in which each clay mix is deposited in layers over a light formwork, such as with the wattle and daub technique. More sustainable solutions also exist for the use of concrete, including shotcrete or sprayed concrete over light formwork composed of fabrics, inflatables or metal meshes. This research explores robotic techniques for the digital fabrication of monolithic earthen shells, with the objective of reformulating the use of clay as a sustainable material to reduce laborious tasks, minimize the use of formwork, and to implement robotic fabrication processes. This unique technique is called "bioshotcrete" and is characterised by an innovative fabrication process of sequential robotic spraying deposition of different natural raw clay mixes over a temporary light formwork. Two case studies are described and analysed featuring two distinctive techniques: clay mixes sprayed with a robotic arm and with a drone. Details are highlighted, and key considerations are identified, in terms of subtle adjustments for the material formulation and application sequences, robotic tooling strategies, and customised robotic actions. This series of experiments was formulated as an ongoing experiment to address challenges related to limitations of reaching distances and lightness of machines to bring on site, and to explore newfound possibilities for aerial deposition techniques using drones. Variations related to Tool/Matter performance (spray velocity and surface adhesion) were explored at each clay mixture iteration. Additional improvements were identified by recent physical tests, such as using the drafts created by the drone helixes to help the drying process at each layer, and additional conclusions establish how this technique is not only shaping new design and digital fabrication processes but envisioning possible future applications and offering new scenarios for sustainable large-scale earthen envelopes.

$$
\begin{gathered}
1 \\
\text { INTRODUCTION }
\end{gathered}
$$

Clay has been used since ancient times as a construction material in vernacular and traditional building practices.
Some techniques like wattle and daub feature intertwined Some techniques like wattle and daub feature intertwined flexible branches as lost formwork that is sequentially coated
by hand with different clay mix layers applied on both sides M. BRAVO / S. CHALTIEL / W. CARAZAS

used for discrete based construction or for building monolithic domes and vaults. The potential to revisit some principles ound in these traditional construction techniques is that when associated with recent design protocols and robotic abrication methods, an unexpected degree of freedom can e unveiled, such as constructing diverse non-regular vaults orking under compression only (Block et al. 2010, 4-13), llowing varying thicknesses and textures from generated ptimized geometries to use minimum material to achieve 

without formwork (DBFL TU Braunschweig, 2018). In addition, drones have been used in construction over recent years mostly to 3D scan construction sites to give feedback information to the performing robot as seen with the recen al. 2018, 248-259). Customised drones were used in the Paint ral fibres textiles such as jute (Chaltiel, Bravo, 2017 94-97).
By Drone project for paint spraying by Carlo Ratti in 2017, For its implementation, a precise process must be followed nd in the weaving drones from Gramazio Kohler Research consisting in the careful formulation of different clay layers together with roboticist Raffaello D'Andrea's group at ETH and in the correct deposition phasing (Chaltiel, Bravo, 2017 Zürich's Institute for Dynamic Systems and Control, who 94-97), aimed at achieving matter/surface adhesion and are researching technological aspects of preprogramed added control in the resulting thicknesses used to construct flights as part of experiments using drones to perform large-scale monolithic earthen domes and shells in only a few non-recording tasks. Research focused on the specific tool/ days. The origin of Bioshotcrete is rooted in the formulation matter interaction could lead to specific architectural forms of a suitable material formulation and application sequence, as highlighted by their resulting non-regular walls in the in- robotic tooling strategy advancements, and the development stallation Remote Material Deposition (Augugliaro et al. 2014, of customised robotic actions.

46-64) with the robotic throwing of small clay lumps. Some of these recent advancements in digital fabrication technologies are opening a fruitful territory for experimenting with novel design processes and construction techniques, offering the ability to reproduce non-regular structures and to optimize shell thicknesses to minimize the amoun

of material used, as featured
Africa (Block et al. 2010 4-13)

This research explores the implementation of mud spraying techniques on light formwork for constructing monolithic earthen shells by using a robotic arm or aerial deposition systems for the spraying strategy (such as drones),
with an emphasis on Tool/Matter calibration for surface adhesion and consistent homogeneous crust forming.

2

ORIGINS OF "BIOSHOTCRETE"

2.1 Paste-like material formulation for additive manufacturing

Clay matter shows clear advantages as a construction material exhibiting environmental benefits such as wide availability as a local natural resource, low entropy, and high inertia. This research proposes a protocol for the sequential robotic deposition of clay mixes by modifying the proportion of each ingredient in the mix, resulting in differaspects. In addition, robotic fabrication using clay mixes requires the careful formulation of materials to ing well to the prerformance in terms of solid crust adhering well to the previous layer or initial surface. Therefore, grains, fbrtion layer has a different composition in which grains, fibres, density, and the proportion of clay which act as a binding agent and water can greatly differ (Fig. 2). Three main types of clay mixtures have been formulated and tested:

1. The liquid layer for the initial spray called "barbotine" forms a homogeneous solid thin crust to replace the initial light formwork. This layer increase adhesion for the following layers, facilitating the removal of the temporary light formwork fabric at the end of the process.

\section{CASE STUDY}

2. Middle layers containing clay water and sands are high in fibres, helping to give thickness without overloading the delicate formwork, and helping to absorb excess moisture. These thick layers provide high coatings contain some natural stabilizing agent such as lime, combined while hot with animal fat thixed in previous layers.

Various physical structures ranging from 1 to $3 \mathrm{~m}$ high and mud sprayed robotically on tense fabric were constructed following a precise material layer formulation (Fig.2) that includes a material composition described in terms of granulometry and proportion of ingredients, as follows:

a- Interface Layer or Barbotine: Composed of clay powder with a grain diameter of

b- Textile reinforcements that are porous with varying mesh densities (such as jute) of $1 \mathrm{~cm}$. min. density that are applied by hand;

c- Layers 2, 3 and 4:1 $\mathrm{U}$ clay $+1 \mathrm{U}$ hard sand [2 $\mathrm{mm}$ diameter] + $1 \mathrm{U}$ water

d- Layers 5, 6 and 7:1 $\mathrm{U}$ clay $+1 \mathrm{U}$ hard sand [4 $\mathrm{mm}$ diameter] + $1 \mathrm{U}$ water;

e- Textile reinforcement layer with a minimum mesh density of $0.5 \mathrm{~cm}$. Applied by hand;

f- Layers 8, 9 and 10: $1 \mathrm{U}$ clay $+1 \mathrm{U}$ hard sand [5 mm diameter] $+1 \mathrm{U}$ water. On the upper layers, differen kinds of stabilizers can be added to the clay mix for waterproofing and to help prevent cracks.

A range of mixes were formulated and implement ed in the featured case studies and will be compared in terms of parameters for matter deposition with good adhesion to the formwork surface. Also, aspects such as composition and sequencing will be further described, including application sequence in phases, as well as material composition in terms of ingredient types, granulometry, proportions of each material, drying times and the use of stabilizers.

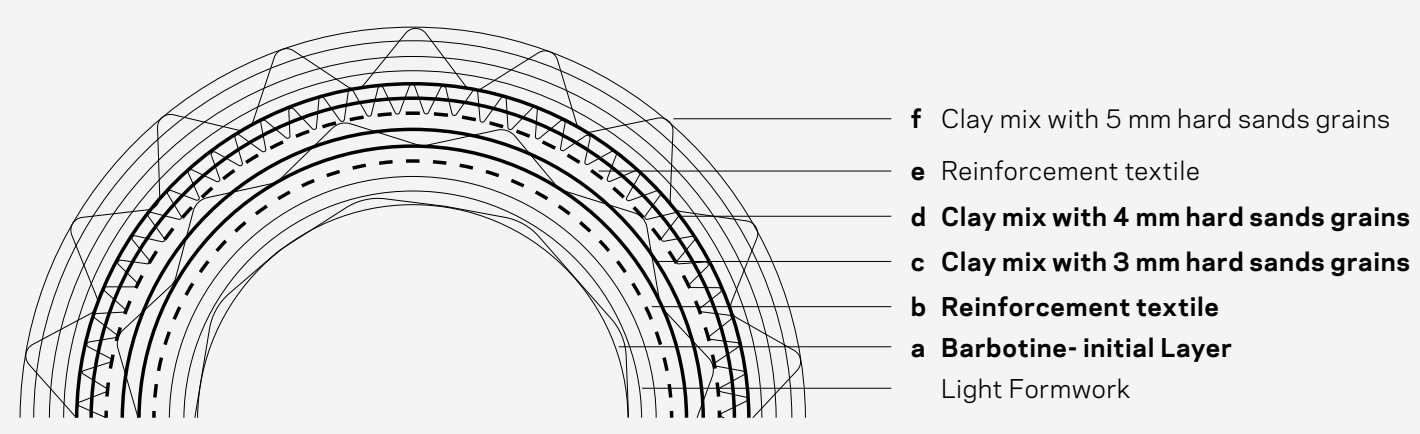

M. BRAVO / S. CHALTIEL / W. CARAZAS self-standing structure is completed.
2.2 Robotic Tooling Strategies

Customised robotic tools to spray different types of ay mixtures over light formwork were identified as relevant cause they eliminate the need for labour intensive scaffoldg, but exposed certain limitations, such as the robotic arm's such as aerial deposition are therefore explored. These early initiatives prove that robotically sprayed clay mixes remain phased robotic construction sequence for clay mixes using robotic arms and drones. The integration between matter nd robotic actions must be carefully choreographed in a rocess defined by the sprayer device, the material container, and the process of continuously feeding the apparatus until

arial container: The first part of the manufacturing process is initiated with the clay mixing with construction equipment (horizontal or "spinner"
mixers). After the mixture is properly combined, it is placed in a container that must be continuously stirred as it had been observed in previous experiences that because clay is a granular material, it tends to adhere to the container walls, causing the mixture to leave a "mouthpiece" with irregular and non-homogeneous edges, creating disparities in the coating.

Material transport: For its deposition, suitable feeding plored:

with several modalities were ex-

1. Robotic arm fitted with a mortar hand sprayer able to carry up to $8 \mathrm{~L}$ of mix attached to a pipe
pressor.

2. Matter deposition with drones in containers with a carrying capacity between 5 to 35 $\mathrm{kg}$ or connected to a ground container using a feeding line.

Sprayer output format: For the discharge, clay material must be in a hydric state compatible to pulverization (viscous). Therefore, as established in previous by a pluri-disciplinary team of designers, engineers an robotic experts, aimed at formulating a more sustainable paste as the matter instead of concrete, smaller equipment

TEMES DE DISSENY \#34 
tests, the machine must be capable of expelling matter with a specific granulometry with all i.e. between clays, silts and fine sands. The type of outlet or "nozzle" could also be adapted in size (diameter) and shape. Therefore, a series of DYI The proposed methodology aims to describe suitable techific uses that niques for the robotic spraying of clay mixes in terms of can be changed according to the design and route material formulation, robotic tools and equipment, and depof the robot, which could be improved and adapted depending on the different forms and structures.

4. The type of deposition apparatus is usually an attachment ment rication methods feature two distinctive robotic fabrication to a robotic arm composed of an end effector Wag- techniques to confirm the validity of the process and to calner Flexio Spray or a mortar sprayer (machine a ibrate the spraying instruments with different kinds of clay sablon), or in the case of drones, a DIY sprayer mixtures. The first case study uses a 6 -axis Kuka robotic or a Wagner Flexio Sprayer.

Each case study features unique robotic tools suitable for robotic arm and aerial drone deposition, including robotic apparatus, material container, material transpor which will be detailed in the case studies.

2.3 Robotic actions for clay spraying

Robotic actions must be carefully calibrated to suit a precise application sequence. Initial tests helped surface to reach of correct matter adhesion to the support shape the nature of the robotic spraying experiments by varying the type of trajectory the tool follows and associating a speed with a specific kind of trajectory. For example, horizontal circular lines need to be done at a speed of 0.5 Name: Clay mixes sprayed with a robotic arm. . and co-pilots for drone experiments.

arm fitted with a heavy paint sprayer to apply different layers of clay mixes over a temporary textile formwork. The second case study takes advantage of the latest drone technology piloted by a professional to carry a container and to deposit layers of different clay mixes over an inflatable formwork as an intent to address the challenges highlighted in case study 1 in terms of the reaching capacity and the use of heavy apparatus that needs to be brought on site. Within each case study, the performance of tool/matter will be 1. The optimum distance between surface and nozzle or drone end of pipe should be between 5 $45 \mathrm{~cm}$. of 5 students each). (IAAC) of Advanced Archi

ent: Industrial Kuka Robotic arm; Wagner heavy

paint sprayer; traditional mortar hand sprayer.
2. Matter Flow Rate should be between 2 Litres and 9 Litres Objective: To build a monolithic earthen shell fitting in a per minute.

3. The Optimum Speed of the Robotic Arm or Drone should be between $0.5 \mathrm{~cm}$ per second and $1 \mathrm{~cm}$ per second 1-metre cube bounding box. A total of five structures were built in one week.

This experiment features a robotic arm with 6 degrees of freedom using only the end effector's last articul of Trajectories Figures: Circular trajectories are tion (Ar6) of the robot (Fig.4). The robotic arm is first fitted
suitable for initial spraying sequences starting
with an off-the-shelf Wagner Flexio sprayer then fitted with a suitable for initial spraying sequences starting with an off-the-shelfWorith from the bottom layers towards the top coats. traditional mortar hand sprayer to deposit the more viscous Vertical depositions must always be done from and high in fibre mixes (Fig.5). Other articula "sins are set by bottom to top at a minimum speed of $0.5 \mathrm{~cm}$ per default to allow the Ar6 trajectories to avoid "singularities", second, while horizontal motions must be done defined as errors in the robotic code where actions cannotbe
for thicker mixes that might contain fibres at a performed, for instance if one part of the robotic arm would for thicker mixes that might contain fibres at a performed, for instance if one part of the robotic arm would
minimum speed of $1 \mathrm{~cm}$ per second. Kuka Prc free software, and the robotic trajectories an These key parameters were formulated based on actions can be set with Grasshopper for Rhino 3D definitions. veral tests and in careful iterative adjustments performed (o match the robotic apparas deposing capabilities, which will be detailed for each case study. A succession of clay mixes was sprayed following a strict deposition sequence detailed in Table 1 . Several stabilisers were tested, typically a highly viscous matter similar to gels or liquid soaps, including a cactus based traditional waterproofing solution that was sprayed successfully with a high matter flow and no detected sprayer blockage. This case study proves that the technique allows CASE STUDY 82
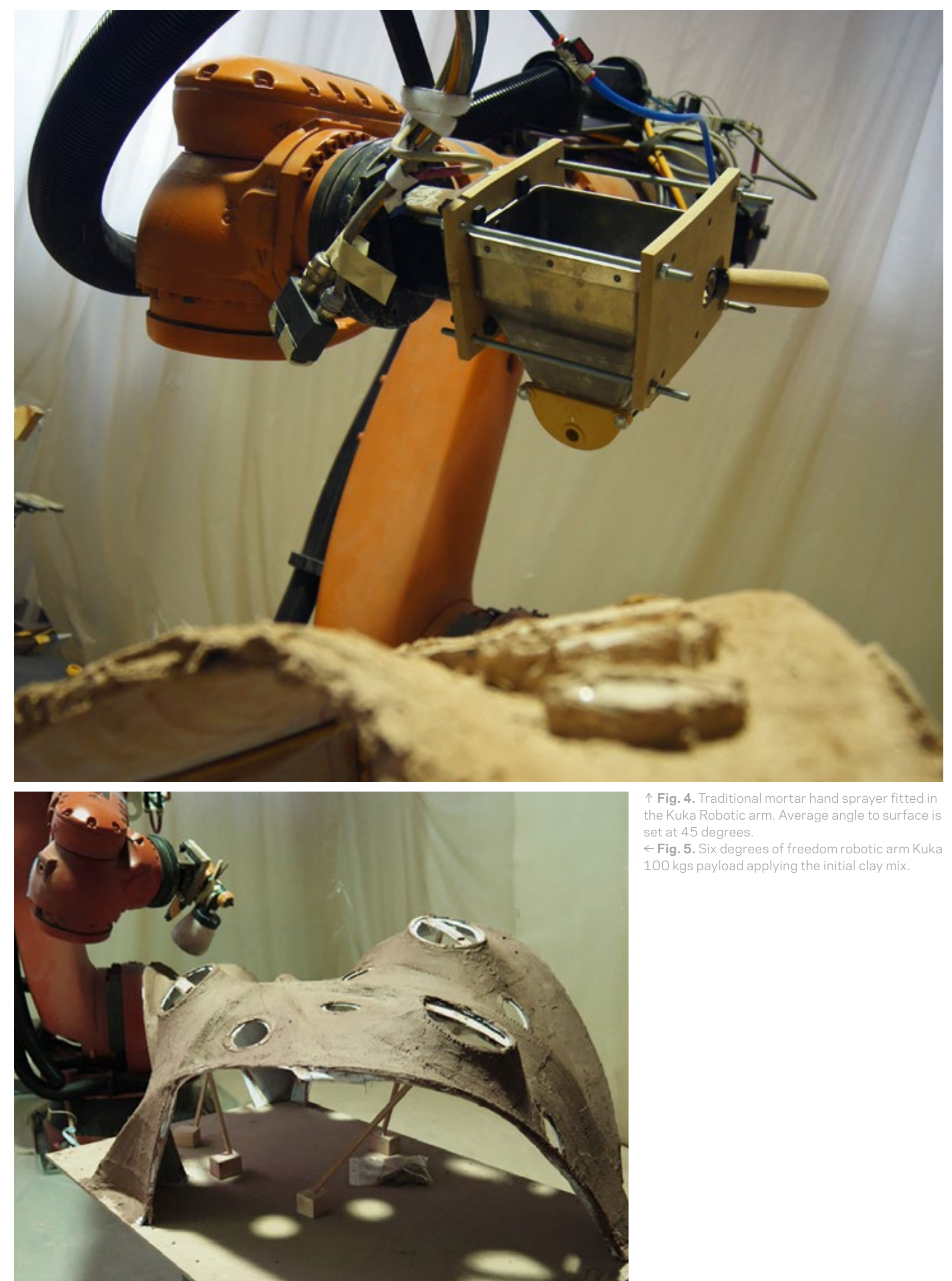

M. BRAVO / S. CHALTIEL / W. CARAZAS

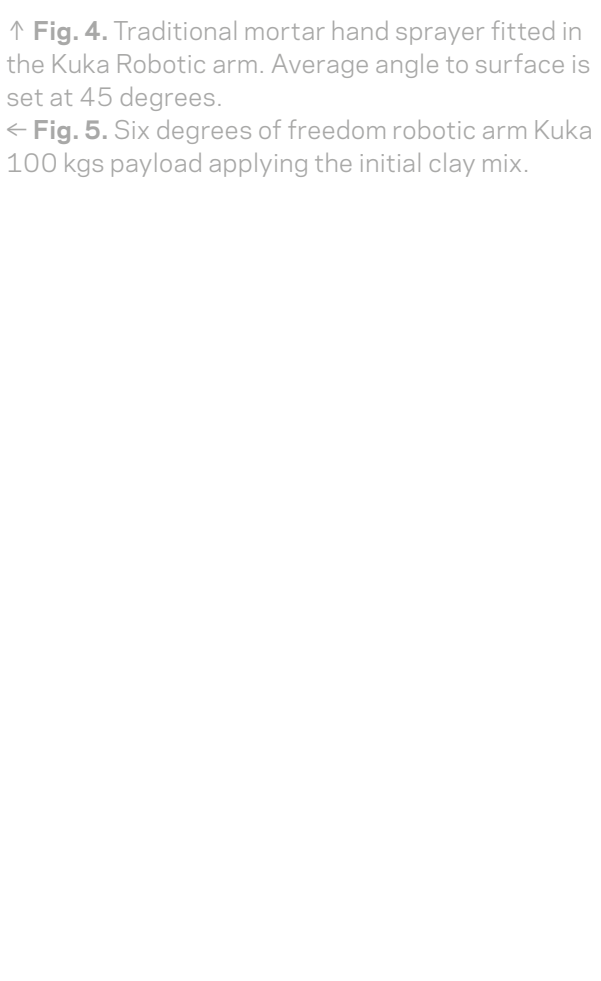

83
TEMES DE DISSENY \#34 
